\title{
Clinical and prognostic features in asymptomatic and symptomatic patients with arteriosclerosis obliterans
}

\author{
Hiromichi Ohsaka, Kazunori Shimada, Kosuke Fukao, Eiryu Sai, Yoshifumi Fukushima, \\ Hiroshi Masuda, Kuniaki Hirose, Takuma Yoshihara, Rie Matsumori, Makoto Hiki, Takashi Kiyanagi, \\ Atsumi Kume, Haruyo Yamashita, Tetsuro Miyazaki, Hiroyuki Daida
}

Department of Cardiology, School of Medicine, Juntendo University, Tokyo, Japan

Email:shimakaz@juntendo.ac.jp

Received 20 November 2011; revised 24 December 2011; accepted 5 January 2012

\begin{abstract}
Background: Patients with arteriosclerosis obliterans (ASO) often have co-existing atherosclerotic diseases. The purpose of this study was to examine the clinical features of patients with ASO, including the overlap of atherosclerotic risk factors, characteristics of cardiovascular events, and clinical prognosis. Method: We enrolled 205 consecutive patients who had ankle brachial index (ABI) of $\leq 0.9$ between January 2008 and December 2009. Fontaine (F) classification and clinical background were evaluated and clinical events including mortality and major adverse cardiocerebrovascular events (MACCEs) were determined. Results: There was a high prevalence of each risk factor. Sixtyfive percent of subjects had three or more of the four overlapping risk factors, including hypertension, diabetes, dyslipidemia, and smoking. After a maximum follow-up of 800 days, the incidence of MACCEs and mortality was $46 \%$ and $10 \%$, respectively. We divided the patients into two groups according to the presence of ASO symptoms (F1 and F2-4) and compared the incidence of events. The incidence of MACCEs and mortality in the F2-4 group was significantly higher than that in the $\mathrm{F} 1$ group $(P=0.048, P=0.044$, respectively). After excluding lower extremity revascularization, coronary artery disease was a common cause of MACCEs, and the mortality rates after MACCEs increased in a stepwise manner according to $F$ classification severity $(P=0.028)$. Conclusion: Patients with ASO had overlapping coronary risk factors and a high incidence rate of cardiovascular events. The incidence of coronary events was common, especially in symptomatic patients, and the mortality rates after MACCEs were high in accordance with $F$ classification severity.
\end{abstract}

Keywords: Arteriosclerosis Obliterans; Fontaine Classification; Risk Factor; Mortality;

Cardiocerebrovascular Event

\section{INTRODUCTION}

The prevalence of atherosclerotic risk factors continues to increase throughout the world, including Japan, because of the spread of western lifestyle habits $[1,2]$. There has been a concomitant increase in atherosclerotic diseases, and heart disease and stroke have become the \#2 and \#3 causes of death in Japan according to 2010 statistics [3]. In addition, the Japanese population is aging rapidly [4]. Therefore, it is believed that the number of patients with arteriosclerosis obliterans (ASO) has been increasing. Epidemiological studies in the general population have shown the incidence of ASO to be $1 \%$ in people aged below $60 \%$ and $3.4 \%$ in people aged 65 or more [5]. Furthermore, the estimated number of patients with ASO in Japan, including asymptomatic patients, ranges from 500,000 to 800,000 [6-8]. Patients with ASO have also been shown to have a high risk of developing other atherosclerotic diseases [9-11]. Although, there have been previous studies of cardiovascular events and clinical prognosis in patients with ASO [9,12,13], it is likely that circumstances regarding the overlapping of various lifestyle-related risk factors and the incidence of cardiovascular events are changing over time. Thus, it is important to examine the clinical and prognostic features of patients with ASO indicating the recent clinical situation of various novel drug treatments for hypertension (HT), diabetes mellitus (DM), and dyslipidemia (DL). In addition, there have been no reports to date on the mortality rates of patients with ASO after the incidence of cardiovascular events.

Thus, the purpose of this study was to examine the clinical features of patients with ASO, including the overlapping of atherosclerotic risk factors, characteristics of cardiovascular events, and clinical prognosis.

\section{METHODS}

\subsection{Subjects}

Two hundred and five consecutive patients with an ankle 
brachial index (ABI) of $\leq 0.9$ measured using the form ABI/PWV (Omron Colin Co., Ltd, Tokyo) were enrolled between January 2008 and December 2009. Patients with acute coronary syndrome (ACS) within the last three months, those hospitalized due to congestive heart failure (CHF), those with active infection and systemic inflamematory diseases, and those with scheduled lower extremeity revascularization were excluded from the study. Subjects were classified according to the Fontaine (F) classification into F1, no symptoms in the lower extremities; F2, intermittent claudication; F3, pain at rest, and F4, skin ulceration and/or necrosis of lower limbs [14]. DL was diagnosed in patients undergoing treatment having total cholesterol (TC) levels of $220 \mathrm{mg} / \mathrm{dL}$ or higher, low-density lipoprotein cholesterol levels (LDL-C) of 140 $\mathrm{mg} / \mathrm{dL}$ or higher, high-density lipoprotein cholesterol (HDL-C) levels of less $40 \mathrm{mg} / \mathrm{dL}$ or lower, or triglycerides (TG) levels of $150 \mathrm{mg} / \mathrm{dL}$ or higher [15]. HT was diagnosed in patients undergoing treatment and having a blood pressure of 140/90 mmHg or higher [16]. DM was diagnosed in patients undergoing treatment and in those with hyperglycemia by two or more tests with blood glucose levels of $200 \mathrm{mg} / \mathrm{dL}$ or higher and an HbA1c reading of $6.5 \%$ or higher (Japan Diabetes Society 6.1\%) [17]. Estimated glomerular filtration rate was calculated for chronic kidney disease (CKD) according to guidelines [18]. Furthermore, antiplatelet agents and prostaglandin properties were also examined in drug treatment histories in addition to the above mentioned drugs. Written informed consent was obtained from all subjects and the ethics committee of the institution approved this study.

\subsection{Major Adverse Cardiocerebrovascular Events (MACCE)}

We observed the clinical course of the subjects for a maximum of 800 days. MACCEs were defined as cardiovascular deaths, non-fatal myocardial infarction, unstable angina, coronary revascularization, admission for stroke, heart failure requiring hospitalization, lower extremity amputation, and lower extremity revascularization.

\subsection{Statistical Analysis}

Student's $t$-test, Welch's $t$-tests, or Mann-Whitney $U$-test was used for comparison of clinical data, and the $\chi^{2}$ test was used for ratio comparison. ANNOVA test was used for comparison of three or more groups. JUMP8.0 MDSU statistical software (SAS Institute, Cary, NC, USA) was used for statistical analysis of survival curves and results were verified using the paired $t$-tests or Wilcoxon test. Data was expressed as mean \pm standard deviation and significant difference determined with a $P<$ 0.05 was considered to be statistically.

\section{RESULTS}

\subsection{Comparison of Clinical Features}

The clinical features of the 205 subjects are shown in Table 1. The average age of the subjects was 71 years \pm 10 years, and $78 \%$ of them were male. They average body mass index was $22.1 \mathrm{~kg} / \mathrm{m}^{2} \pm 3.3 \mathrm{~kg} / \mathrm{m}^{2}$. The prevalence of HT, DM and DL was $84 \%, 54 \%$, and $77 \%$, respectively. Twenty-four percent were current smokers and $44 \%$ were former smokers. Twenty-seven patients had undergone dialysis. The prevalence of myocardial infarction, percutaneous coronary intervention, and coronary aorta bypass grafting was $30 \%, 29 \%$, and $23 \%$, respectively. Percentages of F1, F2, F3, and F4 were 38\%, $44 \%, 12 \%$, and $6 \%$, respectively. Significant differences in ABI $(P<0.001)$, TC $(P=0.045)$, HDL-cholesterol $(P$ $=0.027)$ and prostaglandin administration $(P=0.015)$ were observed among the four groups.

\subsection{Risk Factor Merger Rate and Multiplicity}

Figure 1 shows the rates of overlap among HT, DM, DL and smoking (SM). Overlapping of all four risk factors was observed in $23 \%$, three risk factors in $65 \%$ and two risk factors in $86 \%$ of the subjects. Except a frequent history of SM in males, there was little gender difference in prevalence of the four factors.

\subsection{Clinical Prognosis}

Figure 2 demonstrates the incidence of MACCEs and total mortality. Forty-six percent of subjects suffered a MACCEs with lower extremity revascularization being the most common (39\%), followed by CHF (23\%), CAD (21\%), and cerebrovascular disease (CVD; 11\%). Twentyone patients died (10\%) because of CAD (33\%), CHF (24\%), CVD (19\%). There was a significant difference in the Kaplan-Meier curves of MACCEs between the four F groups. Because there were few F4 subjects, a comparison between the asymptomatic ASO group (F1) and symptomatic ASO group (F2-4) is shown in Figure 3. The F2-4 group had a significantly higher incidence of MA-CCEs than the F1 group (log-rank test, $P=0.048$ ). Similarly, there was a significant difference in KaplanMeier mortality event curves between the four $\mathrm{F}$ groups (logrank test: $P<0.001$, Wilcoxon test: $P<0.001$ ). The F2-4 group had a significantly higher incidence of mortality than the F1 group (log-rank test, $P=0.044$ ).

\subsection{Mortality Rate after Cardiovascular Events}

Figure 4 shows the details of the cardiovascular events occurring in the four $\mathrm{F}$ groups. The proportion of lower extremity revascularization in groups F2, F3 and F4 was $51 \%, 33 \%$, and $80 \%$, respectively. In analysis that excluded lower extremity revascularization, the incidence of CAD and mortality increased with F classification severity. 
Table 1. Patient characteristics. Data are presented as means \pm SDs or percentages. F: Fontaine, HDL: high-density lipoprotein, LDL: low-density lipoprotein, eGFR: estimated glomerular filtration rate, hsCRP: high sensitive C-reactive protein, JDS; Japan Diabetes Society.

\begin{tabular}{|c|c|c|c|c|c|c|}
\hline & Total & F1 & $\mathbf{F} 2$ & F3 & F4 & $\boldsymbol{P}$ \\
\hline Number & 205 & 78 & 90 & 25 & 12 & \\
\hline Age, years & $71 \pm 10$ & $70 \pm 11$ & $72 \pm 9$ & $73 \pm 8$ & $66 \pm 13$ & 0.214 \\
\hline Male (\%) & $160(78)$ & $58(74)$ & $73(81)$ & $20(80)$ & $9(75)$ & 0.745 \\
\hline Body mass index, $\mathrm{kg} / \mathrm{m}^{2}$ & $22.1 \pm 3.3$ & $22.2 \pm 3.5$ & $22.0 \pm 3.3$ & $22.5 \pm 2.8$ & $21.0 \pm 2.6$ & 0.646 \\
\hline Hypertension (\%) & $173(84)$ & $68(87)$ & $73(81)$ & $20(80)$ & $12(100)$ & 0.286 \\
\hline Diabetes mellitus (\%) & $113(55)$ & $41(53)$ & $48(53)$ & $15(60)$ & $8(67)$ & 0.756 \\
\hline Dyslipidemia (\%) & $158(77)$ & $57(73)$ & $73(80)$ & $20(80)$ & $8(67)$ & 0.495 \\
\hline \multicolumn{7}{|l|}{ Smoking history } \\
\hline Current (\%) & $50(24)$ & $14(18)$ & $26(29)$ & $6(24)$ & $4(30)$ & 0.462 \\
\hline Former (\%) & $92(45)$ & $32(41)$ & $42(47)$ & $16(62)$ & $2(18)$ & 0.098 \\
\hline \multicolumn{7}{|l|}{ History of coronary artery disease } \\
\hline Myocardial infarction (\%) & $62(30)$ & $27(33)$ & $24(26)$ & $6(22)$ & $5(45)$ & 0.439 \\
\hline Percutaneous coronary intervention (\%) & $59(29)$ & $22(28)$ & $27(30)$ & $9(37)$ & $1(10)$ & 0.495 \\
\hline Coronary aorta bypass grafting (\%) & $47(23)$ & $21(27)$ & $19(20)$ & $3(11)$ & $4(36)$ & 0.289 \\
\hline \multicolumn{7}{|l|}{ History of peripheral revascularization } \\
\hline Peripheral bypass (\%) & $29(14)$ & $9(11)$ & $18(20)$ & $3(11)$ & $0(0)$ & 0.168 \\
\hline Peripheral intervention (\%) & $55(27)$ & $19(25)$ & $24(27)$ & $12(47)$ & $0(0)$ & 0.018 \\
\hline History of cerebrovascular disease (\%) & $61(30)$ & $28(36)$ & $22(24)$ & $8(32)$ & $3(25)$ & 0.421 \\
\hline Hemodialysis (\%) & $27(13)$ & $8(10)$ & $11(12)$ & $5(20)$ & $3(25)$ & 0.580 \\
\hline Ankle brachial index & $0.70 \pm 0.14$ & $0.75 \pm 0.12$ & $0.66 \pm 0.14 *$ & $0.65 \pm 0.12 *$ & $0.76 \pm 0.13$ & $<0.001$ \\
\hline Brachial-ankle pulse wave velocity, cm/s & $1688 \pm 542$ & $1601 \pm 499$ & $1763 \pm 548$ & $1493 \pm 446$ & $1479 \pm 586$ & 0.233 \\
\hline \multicolumn{7}{|l|}{ Laboratory data } \\
\hline Total cholesterol, mg/dl & $178 \pm 52$ & $173 \pm 34$ & $189 \pm 66$ & $166 \pm 39$ & $157 \pm 41$ & 0.045 \\
\hline Triglyceride, mg/dl & $144 \pm 216$ & $133 \pm 69$ & $171 \pm 321$ & $101 \pm 42$ & $111 \pm 45$ & 0.436 \\
\hline HDL cholesterol, mg/dl & $47 \pm 16$ & $43 \pm 13$ & $50 \pm 17$ & $49 \pm 19$ & $44 \pm 15$ & 0.027 \\
\hline LDL cholesterol, mg/dl & $102 \pm 30$ & $104 \pm 27$ & $105 \pm 30$ & $95 \pm 32$ & $91 \pm 36$ & 0.231 \\
\hline Fasting plasma glucose, mg/dl & $96 \pm 25$ & $101 \pm 27$ & $94 \pm 26$ & $91 \pm 21$ & $92 \pm 17$ & 0.374 \\
\hline HbA1c,\% (JDS) & $\begin{array}{c}6.5 \pm 1.2 \\
(6.1 \pm 1.2)\end{array}$ & $\begin{array}{c}6.5 \pm 1.1 \\
(6.1 \pm 1.1)\end{array}$ & $\begin{array}{c}6.4 \pm 1.0 \\
(6.0 \pm 0.9)\end{array}$ & $\begin{array}{c}6.9 \pm 1.8 \\
(6.5 \pm 1.9)\end{array}$ & $\begin{array}{c}6.5 \pm 1.3 \\
(6.1 \pm 1.3)\end{array}$ & 0.473 \\
\hline eGFR, $\mathrm{ml} / \mathrm{min} / 1.73 \mathrm{~m}^{2}$ & $54 \pm 28$ & $49.5 \pm 26.9$ & $55.6 \pm 26.1$ & $58.8 \pm 30.5$ & $59.6 \pm 43.1$ & 0.373 \\
\hline hsCRP, mg/dl & $0.692 \pm 1.934$ & $0.562 \pm 1.653$ & $0.844 \pm 2.305$ & $0.760 \pm 2.000$ & $0.748 \pm 1.448$ & 0.934 \\
\hline Brain natriuretic peptide, pg/dl & $217 \pm 358$ & $260 \pm 353$ & $192 \pm 386$ & $144 \pm 221$ & $316 \pm 470$ & 0.374 \\
\hline \multicolumn{7}{|l|}{ Medications } \\
\hline Aspirin (\%) & $150(73)$ & $55(71)$ & $68(76)$ & $19(76)$ & $8(64)$ & 0.803 \\
\hline Ticlopidine (\%) & $29(14)$ & $12(16)$ & $14(16)$ & $2(8)$ & $1(9)$ & 0.735 \\
\hline Clopidgrel (\%) & $38(19)$ & $13(17)$ & $15(17)$ & $8(32)$ & $2(18)$ & 0.345 \\
\hline Cilostazol (\%) & $50(25)$ & $14(18)$ & $25(28)$ & $8(32)$ & $3(27)$ & 0.395 \\
\hline Prostaglandin (\%) & $60(29)$ & $12(15)$ & 35 (39) & $11(44)$ & $2(18)$ & 0.009 \\
\hline Calcium channel blockers (\%) & $82(40)$ & $28(36)$ & $39(43)$ & $10(40)$ & $5(45)$ & 0.811 \\
\hline Renin-angiotensin related agents (\%) & $117(57)$ & $48(62)$ & $50(56)$ & $13(52)$ & $6(50)$ & 0.747 \\
\hline Beta blockers (\%) & $74(36)$ & 30 (39) & $26(29)$ & $11(44)$ & $7(55)$ & 0.203 \\
\hline Statin (\%) & $101(49)$ & $33(42)$ & $52(58)$ & $12(48)$ & $4(36)$ & 0.159 \\
\hline Insulin (\%) & $43(21)$ & $15(19)$ & $18(20)$ & $7(28)$ & $3(27)$ & 0.765 \\
\hline
\end{tabular}

$P$ value; ANOVA of continuous variable,$x^{2}$ test of Nominal scales, ${ }^{*} p<0.05$ vs F4: Dunnett test. 


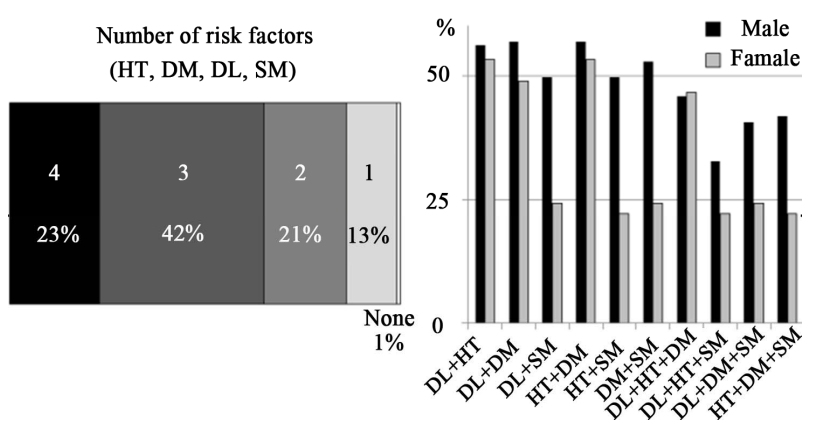

(a)

(b)

Figure 1. Prevalence of overlapping risk factors. There was overlap of all four risk factors (HT, DM, DL, and SM) in 23\% of subjects, three or more risk factors in $65 \%$ of subjects, and two or more risk factors in $86 \%$ of subjects. A trend of more frequent history of SM was evident in males, but there was little gender difference in HT, DM, or DL. HT, hypertension; DM, diabetes mellitus; DL, dyslipidemia; SM, smoking.

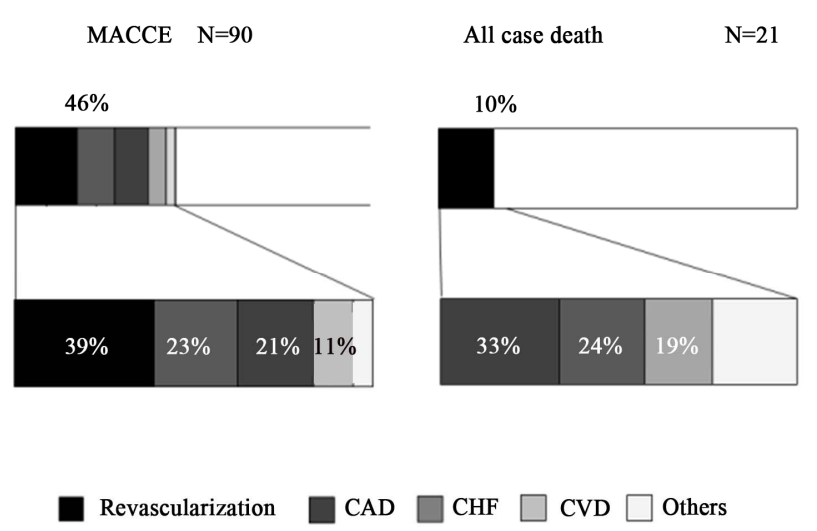

Figure 2. Prevalence and cause of MACCEs and death. Fortysix percent of subjects suffered a MACCE. Among these, lower extremity revascularization was the most common (39\%), followed by CHF (23\%), CAD (21\%) and CVD (11\%). Twentyone patients died (10\%) due to CAD (33\%), CHF (24\%), and CVD (19\%). F, Fontaine; MACCEs, major adverse cardiocerebrovascular events; CAD, coronary artery disease; CHF, congestive heart failure; CVD, cerebrovascular disease.
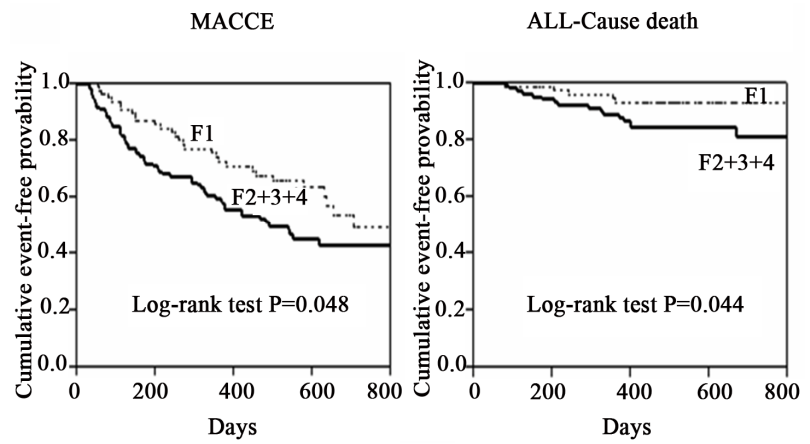

Figure 3. Kaplan-Meyer curves of MACCEs and mortality. The F2-4 groups had a significantly higher incidence of MACCEs and total mortality than that in the F1 group (log-rank test, $P=0.048$ and $P=0.044$ respectively). F, Fontaine; MACCEs, major adverse cardiocerebrovascular events.

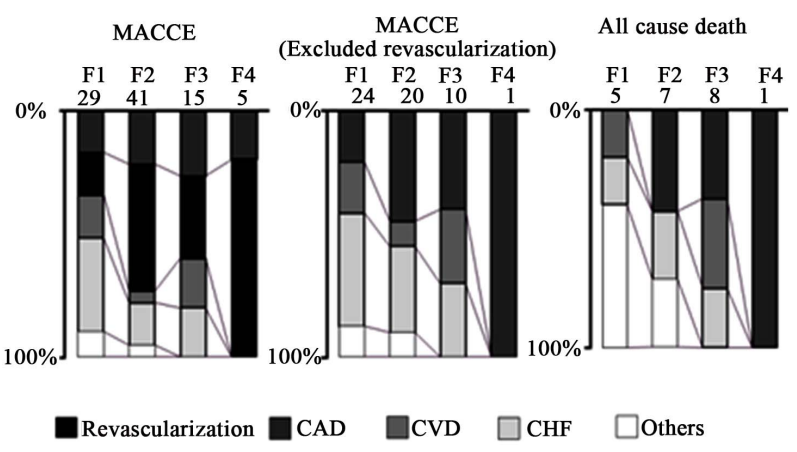

Figure 4. Primary causes of MACCEs and mortality in differrent $\mathrm{F}$ classification after excluding lower extremity revascularization, the incidence of $\mathrm{CAD}$ and mortality increased with $\mathrm{F}$ classification severity. F, Fontaine; MACCEs, major adverse cardiocerebrovascular events; CAD, coronary artery disease; $\mathrm{CHF}$, congestive heart failure; CVD, cerebrovascular disease.

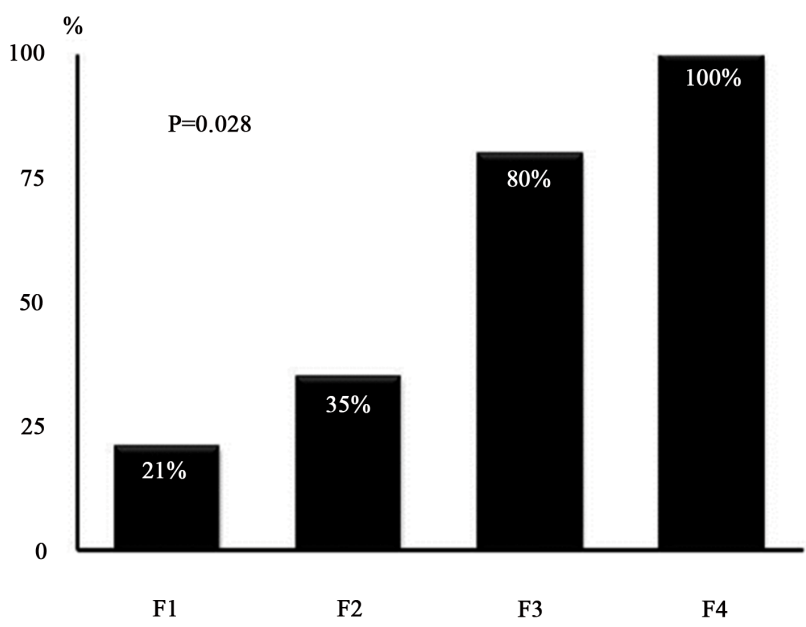

Figure 5. Lethality rates of each $\mathrm{F}$ classification group after cardiovascular events. The mortality rates after the onset of cardiovascular events excluding lower extremity revascularization significantly increased with $\mathrm{F}$ classification severity. F, Fontaine.

Figure 5 shows the mortality rates in the four $\mathrm{F}$ groups after the incidence of cardiovascular events excluding lower extremity revascularization. The mortality rates after cardiovascular events, excluding lower extremity revascularization, significantly increased with $\mathrm{F}$ classification severity $(P=0.028)$.

\section{DISCUSSION}

Patients with ASO had a high prevalence of overlapping coronary risk factors and a high incidence of cardiovascular events. The incidence of coronary events was common, especially in symptomatic patients, and the mortality rates after MACCEs were high in accordance with F classification severity.

In this study, no significant differences in any risk factors were observed among the F groups except for TC 
and HDL-C. However, the prevalence of patients with high TC levels and low HDL-C levels was not necessaryily common in the F3 and F4 groups. Thus, these factors cannot explain why MACCEs and total mortality in the symptomatic F2-F4 groups were higher than those in the F1 group. One possible reason is that the high prevalence of overlapping risk factors may be associated with the onset of clinical events. Indeed, the overlapping rate of three or four of these factors in the MACCEs occurrence and mortality groups was higher compared to the nonMACCEs occurrence and survival groups (data not shown). An increased risk of cardiovascular events in patients with atherosclerotic disease along with overlapping of risk factors has been reported in previous studies $[5,9,19]$. Overlap of risk factors not only accelerates the progression of systemic atherosclerosis and lower extremity peripheral vascular disease but also increases the combined rate of CAD and CVD, resulting in high susceptibility to polyvascular disease. Furthermore, CKD complications including end-stage renal disease and impairment of respiratory function associated with SM are related to the incidence of MACCEs, and also correlated with poor prognosis $[9,11,20]$. The second possible reason involves the recent circumstances of drug treatment. The TASCII guidelines recommended that lipid levels should be controlled by decreasing LDL-C levels to less than $100 \mathrm{mg} / \mathrm{dl}$ (or $70 \mathrm{mg} / \mathrm{dl}$ in patients with CAD), increasing HDL-C levels to $40 \mathrm{mg} / \mathrm{dl}$ or above, and decreasing TG levels to less than $140 \mathrm{mg} / \mathrm{dl}$ [21-25]. Similarly, it is recommended that blood pressure should be controlled at a level of less than 140/90 mmHg (or less than 130/80 mmHg for patients with DM or renal failure) and DM should be controlled at HbA1c levels of less than 7\% [17]. It is necessary to examine the details of the type of drug treatment and dosage in these respective groups in the future. Furthermore, the possibility that the extent of lifestyle improvement in relation to various risk factors is associated with improved prognosis is also a possibility to consider. However, this study did not include any data on nutrition and exercise habits and further examination is needed to investigate these issues.

The cause of death in 7 of 21 subjects who died during this observation period was CAD. Coronary events, excluding lower extremity revascularization, were the common MACCEs. Furthermore, coronary events were also the most common cause of total mortality. It was also clear that the mortality rate after events, excluding lower extremity revascularization, were the common MACCEs. Furthermore, coronary events were also the most common cause of total mortality. It was also clear that the mortality rate after the incidence of cardiovascular events (excluding lower extremity revascularization) increased with F classification severity, which is in agreement with the results of a previous study [26]. However, the pre- sent study demonstrated a novel finding that mortality after cardiovascular events increased a stepwise manner with $\mathrm{F}$ classification severity. As mentioned above, the risk of polyvascular disease including $\mathrm{CAD}$ and the clinical severity of complications may increase along with F classification severity. These combined damages in various organs may be involved in this adverse process. Furthermore, patients with severe ASO could be restricted in their daily living activities, which may mask severe CAD symptoms [27,28]. In addition, it is possible that typical symptoms of CAD may not be readily evident in cases with combined DM or in older subjects $[29,30]$. Therefore, aggressive screening for CAD is required in patients with ASO, especially in F-2 or above.

\section{LIMITATIONS}

This study had several limitations. First, it was a single center study with a small sample size. Second, it is possible that the high multiplicity of risk factors and subsequent high rate of cardiovascular events may be because of selection bias. ABI was measured at the discretion of each physician. However, patients with acute cardiovascular disease and those scheduled for lower extremity revascularization were excluded from this study. Third, hemodialysis patients with a generally poor prognosis were included in this study. We are planning to examine clinical prognosis in patients with ASO and hemodialysis in a relatively large study in the near future. Fourth, the proportion of elderly subjects was relatively high. It may be one of the reasons that BMI levels were relatively low. The combination rate of metabolic syndrome associated with visceral obesity may be different in younger subjects and further studies are needed to assess this possibility.

\section{CONCLUSION}

Patients with ASO had high prevalence of overlapping coronary risk factors and a high incidence ate of cardiovascular events. Such cases with combined defects were example of so-called polyvascular disease, and therefore, required comprehensive management of risk factors. In addition, the incidence of coronary events was common in symptomatic patients and the mortality rates after MACCEs were also high in accordance with F classification severity. Thus, aggressive screening for CAD is required in patients with ASO, especially those in symptomatic for ASO.

\section{REFERENCES}

[1] Thomas, R., Dawber, F.E., George, V.M., et al. (1957) Coronary heart disease in the Framingham study. American Journal of Public Health, 47, 4-24.

doi:10.2105/AJPH.47.4_Pt_2.4 
[2] Takeuchi, H., Saitoh, S., Shimamoto, K., et al. (2005) Metabolic syndrome and cardiac disease in Japanese men: Applicability of the concept of metabolic syndrome defined by the National Cholesterol Education ProgramAdult Treatment Panel III to Japanese men-The Tanno and Sobetsu Study. Hypertens Research, 28, 203-208. doi:10.1291/hypres.28.203

[3] Health and Welfare Statistics Association, Japan (2010/ 2011) Journal of Health and Welfare statistics, 58, 56.

[4] Health and Welfare Statistics Association Japan (2009) Journal of Health and Welfare statistics, 56, 50-54.

[5] Tadashi, F., Shigeyuki, S., Kazuaki, S., et al. (2004) Prevalence of asymptomatic arteriosclerosis obliterans and its relationship with risk factors in inhabitants of rural communities in Japan: Tanno-Sobetsu study. Atherosclerosis, 177, 83-88. doi:10.1016/j.atherosclerosis.2004.05.028

[6] Yamazaki, T., Goto, S., Ikeda, Y., et al. (2007) Prevalence, awareness and treatment of cardiovascular risk factors in patients at high risk of atherothrombosis in Japan. Circulation Journal, 71, 995-1003. doi:10.1253/circj.71.995

[7] Hietanen, H., Pääkkönen, R. and Salomaa, V. (2008) Ankle blood pressure as a predictor of total and cardiovascular mortality. BMC Cardiovascular Disorders, 8, 3. doi:10.1186/1471-2261-8-3

[8] McDermo, T.T., Greenland, P., Criqui, M.H., Dolan, N.C., et al. (2001) Leg symptoms in peripheral arterial disease: Associated clinical characteristics and functional impairment. The Journal of the American Medical Association, 286, 1599-1606. doi:10.1001/jama.286.13.1599

[9] Norgren, L., Hiatt, W.R., Fowkes, F.G., et al. (2007) Inter-society consensus for the management of peripheral arterial disease (TASC II). Journal of Vascular Surgery, 45, S5-S67. doi:10.1016/j.jvs.2006.12.037

[10] Bhatt, D.L., Steg, P.G., Wilson, P.W., et al. (2006) International prevalence, recognition, and treatment of cardiovascular risk factors in outpatients with atherothrombosis. The Journal of the American Medical Association, 295, 180-189. doi:10.1001/jama.295.2.180

[11] Hertzer, N.R., Beven, E.G., Maljovec, L.C., et al. (1984) Coronary artery disease in peripheral vascular patients. A classification of 1000 coronary angiograms and results of surgical management. Annals of Surgery, 199, 223-233. doi:10.1097/00000658-198402000-00016

[12] Fowkes, F.G., Murray, G.D., McDermott, M.M., et al. (2008) Ankle brachial index combined with Framingham Risk Score to predict cardiovascular events and mortality. The Journal of the American Medical Association, 300, 197-208. doi:10.1001/jama.300.2.197

[13] Hamai, K., Hiasa, Y., Mori, H., et al. (1989) Correlation of arteriosclerosis obliterans of the lower extremity with coronary artery disease. Journal of Cardiology, 19, 103112.

[14] Sacks, D. (2000) Trans-Atlantic Inter-Society Consensus (TASC) working group. Journal of Vascular Surgery, 31, S5-S44, S54-S74, S77-S122.

[15] Japan Atherosclerosis Society (2008) Treatment guide for dyslipidaemia.

[16] The Japanese Society of Hypertension (2009) Treatment guide for hypertension.

[17] Japan Diabetes Society (2010) Treatment guide for diabetes.

[18] Japanese Society of Nephrology (2007) Clinical practice guidebook for diagnosis and treatment of chronic kidney disease.

[19] Alberts, M.J., Bhatt, D.L., Steg, P.G., et al. (2009) Threeyear follow-up and event rates in the international Reduction of atherothrombosis for continued health registry. European Heart Journal, 30, 2318-2326. doi:10.1093/eurheartj/ehp355

[20] Hirsch, A.T., Haskal, Z.J., Riegel, B., et al. (2006) ACC/ AHA 2005 Practice Guidelines for the management of patients with peripheral arterial disease (lower extremity, renal, mesenteric, and abdominal aortic). Circulation, 113, e463. doi:10.1161/CIRCULATIONAHA.106.174526

[21] Marcon, G., Barbato, O., Zolli, M., et al. (1991) Unecessary arterial Doppler examination of the legs. Clinical decision rules may help? Quality Assurance Health Care, 3, 115-122.

[22] Sanderson, K.J., van Rij, A.M. and Sutherland, W.H. (1995) Lipid peroxidation of circulating low density lipoproteins with age, smoking and in peripheral vascular disease. Atherosclerosis, 118, 45-51. doi:10.1016/0021-9150(95)05591-J

[23] Kinjo, K., Sato, H., Hori, M., et al. (2005) Impact of smoking status on long-term mortality in patient with acute myocardial infarction. Circulation Journal, 69, 712. doi:10.1253/circj.69.7

[24] Ogata, N., Yamamoto, H., Miyamoto, E., et al. (2000) Involvement of protein kinase $\mathrm{C}$ in superoxide anion-induced activation of nuclear factor-kappa B in human endothelial cells. Cardiovascular Research, 45, 513-521. doi:10.1016/S0008-6363(99)00364-8

[25] Johnson, C.L., Rifkind, B.M., Lippel, K., et al. (1993) Declining serum total cholesterol levels among US adults. The national health and nutrition examination surveys. The Journal of the American Medical Association, 269, 3002-3008. doi:10.1001/jama.1993.03500230084034

[26] Shigematsu, H., Nishibe, T., Matsumoto, T., et al. (2010) Three-year cardiovascular events and disease progress in patients with peripheral arterial disease: Results from the Japan Medication Therapy for Peripheral Arterial Disease (J-METHOD). International Angiology, 29, 2-13.

[27] Hiroyuki, N., Hiroyasu, I., Akiko, T., et al. (2005) Walking and sport participation and mortality from coronary heart disease and stroke. Journal of American College of Cardiology, 46, 1761-1767. doi:10.1016/j.jacc.2005.07.038

[28] Staessen, J.A., Gasowski, J., Wang, J.G., et al. (2000) Risks of untreated and treated isolated systolic hypertension in the elderly: Meta-analysis of outcome trials. Lancet, 355, 865-872. doi:10.1016/S0140-6736(99)07330-4

[29] Diehm, C., Allenberg, J.R., Trampisch, H.J., et al. (2009) Mortality and vascular morbidity in older adults with As- 
ymptomatic versus symptomatic peripheral artery disease. Circulation, 120, 2053-2061.

doi:10.1161/CIRCULATIONAHA.109.865600

[30] Ariyo, A., Haan, M., Tangen, C.M., et al. (2000) Depres- sive symptoms and risks of coronary heart disease and mortality in elderly Americans. Circulation, 102, 17731777. 